\title{
Correspondence
}

\section{Engaging with the out-patient clinic: don't throw the baby out with the bath water}

I could not have been alone among the readers of APT to have returned home after a hard day's toil in my out-patient clinic to be irritated to read that my patients and I had been wasting our time. Killaspy (2007) makes some bold statements about out-patient psychiatric care, casting doubt on the relevance of out-patient clinics to contemporary psychiatric practice. This theme is taken on and broadened by Harrison (2007) in a somewhat uncritical review of changes to the role of the consultant (adult) psychiatrist over the past decade, particularly in the light of New Ways of Working and the functionalised model of adult mental health services.

The argument goes something like this: contemporary services are comprised of multidisciplinary teams whose members have an increasing range of generic competencies that are together providing secondary mental healthcare to people with severe mental illness under the auspices of the care programme approach. Generic competencies include skills in the assessment and 'signposting' of people referred to services who do not have a severe mental illness, and risk assessment and care coordination of those taken onto the case-load who do. Non-medical practitioners are increasingly developing traditional medical competencies, including independent prescribing. The Mental Health Act 2007 introduces the 'responsible clinician', thus breaking the monopoly of the doctor in having charge of the care of the involuntary psychiatric patient that has been effectively in place for 200 years. Within this brave new world the out-patient clinic becomes an irrelevance as, perhaps, soon will the adult psychiatrist. This scenario has already been played out in slightly different ways within learning (intellectual) disability and child and adolescent psychiatry, disciplines that remain in mysteriously good health.

There are a few unfortunate facts that get in the way of this, for adult psychiatrists at least, somewhat depressing vision. First, as Killaspy (2007) notes, although the majority of people who are on the current case-load of community mental health services have demonstrable severe mental illnesses (a code word for psychotic disorders), the majority of referrals for assessment and treatment to secondary services present with other problems that are often conceptually more complex than the psychoses. (The same is true for the in-patient population: there are more admissions of people with non-psychotic disorder, although psychosis accounts for more beddays overall.)
Second, for many services in inner-city areas the available care coordination capacity cannot manage everyone with a diagnosis of psychosis, let alone those cycling through the system with non-psychotic disorders, many of whom are in fact both disturbed and 'risky'.

Third, current training reliably provides psychiatrists with set of skills that is unique in the mental health workforce, particularly in terms of assessment, formulation, diagnosis and psychopharmacology. (It is surely unlikely that a brief protocol-driven course on prescribing, as described by Baldwin \& Kosky (2007), will allow a nurse-prescriber to dabble in the muddy waters of off-label prescribing.)

Fourth, there is continuing demand from referrers, patients and carers for out-patient services. In my own service I could comfortably run additional clinics, to which patients and carers would turn up (helped by our local practice of telephone reminders, which are effective, greatly appreciated by the patients and leave me with distressingly little unstructured time, pace Killaspy) and my work quite appropriately forms part of our trust's activity-based contract. Officebased psychiatric practice flourishes throughout the advanced world and (I understand) private practice is alive and well in the UK.

This is not to argue against the importance of multidisciplinary team-working, of which I am a strong advocate (Holloway \& Chorlton, 2007), nor to suggest that the traditional roles that psychiatrists hold are theirs of right. However, we need a much more nuanced discussion about the future role of psychiatrists within adult mental health services than offered by Killaspy (2007) and Harrison (2007). This requires both firmer conceptual analysis, particularly in relation to the appropriate care pathways that should be provided for people with suspected mental illness, and a lot more empirical work on a neglected aspect of care. It's surely premature to throw out the out-patient baby as we modernise the mental health bath water.

\section{Declaration of interest}

I have throughout my career spent a lot of my time seeing out-patients in clinics.

Baldwin, D. S. \& Kosky, N. (2007) Off-label prescribing in psychiatric practice. Advances in Psychiatric Treatment, 13, 414-422.

Harrison, J. (2007) The work pattern of consultant psychiatrists. Revisiting: How consultants manage their time. Advances in Psychiatric Treatment, 13, 470-475.

Holloway, F. \& Chorlton, C. (2007) Multidisciplinary teams. In Management for Psychiatrists (3rd edn) (Eds D. Bhugra, S. Bell \& A. Burns), pp. 99-115. RCPsych Publications. 
Killaspy, H. (2007) Why do psychiatrists have difficulty disengaging with the out-patient clinic? Invited commentary on: Why don't patients attend their appointments? Advances in Psychiatric Treatment, 13, 435-437.

Frank Holloway Consultant Psychiatrist, South London and Maudsley NHS Foundation Trust, Bethlem Royal Hospital, Beckenham, Kent, UK (email: f.holloway@iop.kcl.ac.uk)

doi: 10.1192/apt.14.2.159

\section{Triangulating views on antipsychotics}

The article by David Cunningham Owens (2008) is one of the most thoughtful, stimulating, witty and clinically relevant that $A P T$ has published. It urges us to reconsider our views on what we call atypical antipsychotics. As an authority on extrapyramidal symptoms, he describes how the absence of parkinsonian side-effects may endow an antipsychotic with other advantages, including lower risks of depression and cognitive impairment, and of worsening negative and perhaps also positive symptoms of schizophrenia. There is no doubt that haloperidol (widely used in clinical trials as a comparator) can be made to appear very inferior, by prescribing it without prophylactic anticholinergic medication, and 'efficacy trials' in which this is done carry a bias against the classical antipsychotic. Owens is not surprised that 'effectiveness studies' such as CATIE fail to show consistent advantages for newer (atypical) antipsychotics in maintenance treatment for schizophrenia.

It would be a mistake to interpret his article as undermining the conclusion that the atypicals represent a therapeutic advance, or to support a conspiratorial view of the pharmaceutical industry. Advances in the treatment of psychosis and severely disturbed behaviour have come very slowly over 150 years, from the use of bromide salts (1857) and sedatives (chloral in 1869, barbiturates from 1905, antihistamines in the 1940s and benzodiazepines from 1961). It was the fortuitous discoveries of the properties of lithium from 1948 and chlorpromazine (a product of the antihistamine industry) from 1952 that represent the beginnings of modern psychotherapeutics. Naturally, drug innovators such as Paul Janssen (1926-2003), who discovered haloperidol (1958), wanted to produce an antipsychotic that would have fewer extrapyramidal side-effects and greater efficacy than haloperidol and the other older drugs. They saw the opportunity to do this, first by selective blockade of subtypes of dopamine receptors (the benzamide drugs), and later by modifying the structure of clozapine (e.g. olanzapine and quetiapine) and by attempting to mimic its pharmacological actions, especially blockade of serotonin (5-HT) receptors (e.g. risperidone). Thus, the atypicals represent the application of neuroscientific knowledge and logic to drug development. What this has produced for clinicians (treating some of the most devastating human disorders) is a range of therapeutic options with a variety of different side-effects and possibly some differences in efficacy.

The effectiveness studies discussed by Owens involved randomisation of patients to receive one of several possible drugs. This is an unnatural procedure that avoids the crucial step in which a clinician discusses the available drugs with the patient and then decides which might best suit their needs.

The findings of CATIE (funded independently of industry) do suggest that some atypicals are more likely than other drugs to be continued, for reasons of both efficacy and individual side-effects, although the differences are relatively small.

A third angle from which to view treatments is that of the 'observational study', in which a large cohort of patients is allocated a treatment, chosen by the clinician. These studies tend to confirm that the individual properties of different drugs (sedation, weight gain and metabolic effects, endocrine and sexual side-effects, and extrapyramidal side-effects) do occur in the real-world setting as predicted by the efficacy trials. Such studies tend to be sponsored by the industry and therefore to attract more scepticism. However, the findings should be included in a 'triangulated' view of the role of atypical antipsychotics. This combined information is the basis on which the clinician can make the individualised risk/benefit appraisal recommended by Owens and illustrated in his Fig. 2.

The industry has been richly rewarded for its investment in research in neuroscience and psychosis, and it will need this success to make the further investments that are required to explore the wealth of information that is arising from the basic neurosciences. For example, the exploration of glutamate (and the phencyclidine-PCP-model of psychosis) and endocannabinoids and their interaction with dopamine are tantalising subjects for therapeutic research and development. Moreover, the function and pharmacology of dopamine pathways has probably much more to tell us about psychosis, mood disorders and addictions.

\section{Declaration of interest}

J.C. has provided advice and lectures at meetings sponsored by the manufacturers of several atypical antipsychotics, including those mentioned here. He has met Paul Janssen and reviewed his biography.

Owens, D. C. (2008) How CATIE brought us back to Kansas: a critical re-evaluation of the concept of atypical antipsychotics and their place in the treatment of schizophrenia. Advances in Psychiatric Treatment, 14, 17-28.

John Cookson Consultant in General Adult Psychiatry, Royal London Hospital and East London Foundation Trust, London, UK (email: cookson-psychiatry@boltblue.com)

doi: 10.1192 /apt.14.2.160 